\title{
Lateralization for dynamic facial expressions in human superior temporal sulcus
}

\author{
François-Laurent De Winter ${ }^{\mathrm{a}, \mathrm{b}, *}$, Qi Zhu ${ }^{\mathrm{c}}$, Jan Van den Stock ${ }^{\mathrm{a}, \mathrm{b}}$, Koen Nelissen ${ }^{\mathrm{c}}$, Ronald Peeters ${ }^{\mathrm{d}, \mathrm{e}}$, \\ Beatrice de Gelder ${ }^{\text {a,f,g }}$, Wim Vanduffel ${ }^{\text {c,h,i }}$, Mathieu Vandenbulcke ${ }^{\mathrm{a}, \mathrm{b}}$
}

a Research Group Psychiatry, Department of Neurosciences, KU Leuven, Herestraat 49, 3000 Leuven, Belgium

b Old Age Psychiatry Department, University Hospitals Leuven, Herestraat 49, 3000 Leuven, Belgium

c Laboratory for Neuro- and Psychophysiology, Department of Neurosciences, KU Leuven, Herestraat 49, 3000 Leuven, Belgium

' Department of Radiology, UZ Leuven, Herestraat 49, 3000 Leuven, Belgium

e Department of Imaging \&' Pathology, KU Leuven, Herestraat 49, 3000 Leuven, Belgium

${ }^{\mathrm{f}}$ Department of Cognitive Neuroscience, Maastricht University, P.O. Box 616, 6200 MD Maastricht, The Netherlands

${ }^{g}$ Laboratory for Cognitive and Affective Neuroscience, Tilburg University, P.O. Box 90153, 5000 LE Tilburg, The Netherlands

${ }^{\text {h }}$ Massachusetts Gen. Hosp., Athinoula A. Martinos Ctr. for Biomed. Imaging, 149 Thirteenth Street, Suite 2301 Charlestown, MA, USA

${ }^{i}$ Dept. of Radiology, Harvard Med. Sch., Boston, MA, USA

\section{A R T I C L E I N F O}

\section{Article history:}

Accepted 8 November 2014

Available online $\mathrm{xxxx}$

\section{Keywords:}

Lateralization

Dynamic facial expressions

Comparative

fMRI

\begin{abstract}
A B S T R A C T
Most face processing studies in humans show stronger activation in the right compared to the left hemisphere. Evidence is largely based on studies with static stimuli focusing on the fusiform face area (FFA). Hence, the pattern of lateralization for dynamic faces is less clear. Furthermore, it is unclear whether this property is common to human and non-human primates due to predisposing processing strategies in the right hemisphere or that alternatively left sided specialization for language in humans could be the driving force behind this phenomenon. We aimed to address both issues by studying lateralization for dynamic facial expressions in monkeys and humans. Therefore, we conducted an event-related fMRI experiment in three macaques and twenty right handed humans. We presented human and monkey dynamic facial expressions (chewing and fear) as well as scrambled versions to both species. We studied lateralization in independently defined face-responsive and face-selective regions by calculating a weighted lateralization index (LIwm) using a bootstrapping method. In order to examine if lateralization in humans is related to language, we performed a separate fMRI experiment in ten human volunteers including a 'speech' expression (one syllable non-word) and its scrambled version.

Both within face-responsive and selective regions, we found consistent lateralization for dynamic faces (chewing and fear) versus scrambled versions in the right human posterior superior temporal sulcus (pSTS), but not in FFA nor in ventral temporal cortex. Conversely, in monkeys no consistent pattern of lateralization for dynamic facial expressions was observed. Finally, Llwms based on the contrast between different types of dynamic facial expressions (relative to scrambled versions) revealed left-sided lateralization in human pSTS for speech-related expressions compared to chewing and emotional expressions.

To conclude, we found consistent laterality effects in human posterior STS but not in visual cortex of monkeys. Based on our results, it is tempting to speculate that lateralization for dynamic face processing in humans may be driven by left-hemispheric language specialization which may not have been present yet in the common ancestor of human and macaque monkeys.
\end{abstract}

(c) 2014 Elsevier Inc. All rights reserved.

\section{Introduction}

It is generally assumed that faces are processed asymmetrically in the human brain yet it remains unclear whether lateralization for faces is dependent on stimulus type, regionally selective and human-

\footnotetext{
* Corresponding author at: Research Group Psychiatry, Department of Neurosciences, KU Leuven, Herestraat 49, Box 1027, 3000 Leuven, Belgium.

E-mail address: francoislaurent.dewinter@med.kuleuven.be (F.-L. De Winter).
}

specific. It needs to be noted that lateralization for faces is a debated question in comparative neuroscience. Some argue that hemispheric specialization for facial expressions emerged in parallel with lefthemispheric specialization in verbal communication and is indeed a human property (Overman and Doty, 1982; Corballis et al., 2000), whereas others propose that lateralization was already present earlier in primate ancestors because of predisposing properties of the right hemisphere (Hamilton and Vermeire, 1988; Vallortigara et al., 1999; Zangenehpour and Chaudhuri, 2005). Split-field (Ellis and Shepherd, 1975; Broman, 1978; Reynolds and Jeeves, 1978) and brain lesion 
studies (Sergent and Signoret, 1992; De Renzi et al., 1994; Wilkinson et al., 2009; Busigny et al., 2010) have been the major source of evidence for a privileged role of the right hemisphere in face processing in humans, whereas similar studies in monkeys have yielded conflicting results (Overman and Doty, 1982; Hamilton and Vermeire, 1988; Vermeire and Hamilton, 1998). In chimpanzees results are not conclusive either with a left visual field superiority in processing chimeric faces of humans only and of human and chimpanzee faces (Morris and Hopkins, 1993; Dahl et al., 2013) whereas no such lateralization could be found in a match-to-sample task (Plotnik et al., 2003).

Most of the imaging studies that reported laterality effects for face processing used static faces and focused on the FFA (Dien, 2009). Laterality effects for dynamic faces have not yet been explicitly addressed, despite important differences in neural processing between dynamic and static faces. For instance, STS responds much stronger to dynamic than to static faces and even includes areas that respond selectively to dynamic faces (Pitcher et al., 2011) (see also de Gelder and Van den Stock (2010) for an overview of functional imaging studies using dynamic facial expressions). Furthermore, although stronger activations have been reported in right compared to left human STS, rigorous statistical methods have not been used to investigate lateralization for dynamic faces (e.g. Foley et al. (2012)). Also, recent comparative studies suggested that the specialization of the STS for dynamic facial expressions is stronger in humans than monkeys (Zhu et al., 2013; Polosecki et al., 2013). If the human or hominoid brain developed specialization for dynamic faces and lateralization occurs at the same level, this may imply that lateralization for dynamic faces is also a human-unique property.

The cognitive demands that are associated with behavioral testing hamper comparisons of species that differ importantly in cognitive abilities. Neuroimaging studies on the other hand provide a means of studying lateralized effects directly without a specific task. So far, however, comparative brain imaging studies have not explicitly addressed lateralization of dynamic face processing (Tsao et al., 2003; Pinsk et al., 2009; Polosecki et al., 2013). If language development and specialization in verbal communication are the driving forces behind lateralization for dynamic face processing in humans, one would expect the largest effects in homotopical areas relative to the classical language areas, such as Wernicke's area. Also one would predict that facial gestures that map upon lexical representations, such as speech, would be coded differently compared to non-verbal expressions.

In the present fMRI study we aimed to address three questions. Is lateralization for dynamic faces present in the human brain, and if so where? Is lateralization for dynamic faces a unique property of the human brain? Does lateralization depend on the type of facial gesture, linguistic or emotional? During event-related fMRI, we presented dynamic facial expressions (both chewing and fearful faces), as well as their spatiotemporally mosaic scrambled versions, to humans and macaques. In a separate experiment, we also presented dynamic faces producing speech to humans, in the absence of auditory stimuli. We studied lateralization not only in regions that were responsive to dynamic faces but also in face-selective regions defined by an independent localizer experiment. Lateralization was determined by calculating laterality indices (LIs) using a bootstrapping method within the LI toolbox for SPM8 (Wilke and Schmithorst, 2006; Wilke and Lidzba, 2007). This method yields a robust mean LI-value, with a minimum and a maximum $\mathrm{LI}$ between -1 and 1 indicating right or left hemispheric specialization respectively, while limiting the influence by statistical outliers.

\section{Methods}

Subjects

Twenty healthy volunteers ( 12 female, $22-34$ years old) and three healthy male rhesus monkeys (Macaca mulatta; 5-7 kg, 4-5 years old) participated in Experiment 1 with dynamic human and monkey facial expressions (chewing and fear). Ten human subjects (6 male, 23-36 years old; 8 of which also participated in the first experiment) participated in Experiment 2 with only human dynamic faces but with speech expressions added. A localizer scan to determine face-selective areas was obtained from the latter 10 subjects and the 3 monkeys. For one of the monkeys, the data from the localizer experiment was discarded because of technical problems. The experiments were approved by the ethical committee of the University of Leuven and all human participants gave written informed consent. All human subjects were righthanded as assessed through the Edinburgh Handedness Inventory (mean for 20 subjects of Experiment 1 was 0.92; mean for 10 subjects of Experiment 2 was 0.97). All subjects had normal or corrected-tonormal visual acuity.

Stimuli

\section{Human and monkey dynamic faces}

Movie clips acquired from six professional human male actors and six male monkeys were used for each type of expression in the dynamic face experiments. The chewing and fearful expressions used in Experiment 1 have been described elsewhere (Zhu et al., 2013). The speech stimuli (Experiment 2) consisted of the neutral pronunciation of a one-syllable non-word, but only the visual component of the speech stimuli was used in the experiment. Construction of the stimuli was exactly as described in Zhu et al. (2013): All stimuli were frontal view color movie clips with external face contours removed and mean luminance equalized. Expressions were gaze-averted but with heads fixed. Mirror-reversed versions of each movie clip were also created to control for eye-gaze direction, head orientation and movement asymmetries. Spatiotemporally scrambled control stimuli were generated in which the facial shape information and the dynamic expressions were removed while the low-level motion information from the original clips was retained. Scrambled stimuli were created by applying a temporally scrambled flow field of each movie clip to the mosaic-scrambled start image of the original sequence. The mosaic scrambling was accomplished by dividing the image into a $32 \times 32$ grid and shuffling the positions of the grid elements. The flow field of the original movie clips was calculated using an optic flow estimation algorithm developed by Papenberg et al. (2006), then temporally scrambled by spatially dividing the flow field into an $8 \times 8$ grid and shuffling the frames differently for each grid across temporal blocks with five frames for each block. Fig. 1A gives an illustration of each stimulus type.

\section{Localizer experiment}

Six object categories, each containing 20 static achromatic images, were presented to both humans and monkeys during scanning. These categories included human and monkey faces, headless human bodies and two categories of inanimate manmade objects with different mean aspect ratios (objects $\mathrm{H}$ and objects $\mathrm{M}$ ) (see Popivanov et al. (2012) for further description and illustration of all stimuli). Mosaicscrambles were created by spatially scrambling one category of inanimate manmade objects (i.e. objects $\mathrm{M}$ ). The scrambling was accomplished by dividing the image into a $38 \times 38$ grid and shuffling the positions of the grid within a rectangular area bordering the original object. The mean luminance was equated across stimuli. All stimuli were embedded in a random-noise background having the same luminance as the images. The noise background filled the entire screen.

\section{Experimental design}

\section{Human and monkey dynamic faces}

An event-related design was used in Experiments 1 and 2 (Fig. 1B). Every movie clip was presented once for $2 \mathrm{~s}$, followed by a $2.5 \mathrm{~s}$ to $3.5 \mathrm{~s}$ inter-stimulus interval displaying only the grid. Twelve nulltrials with only the grid presented for $4.5 \mathrm{~s}$ to $5.5 \mathrm{~s}$ were randomly 


\section{Experiment 1}

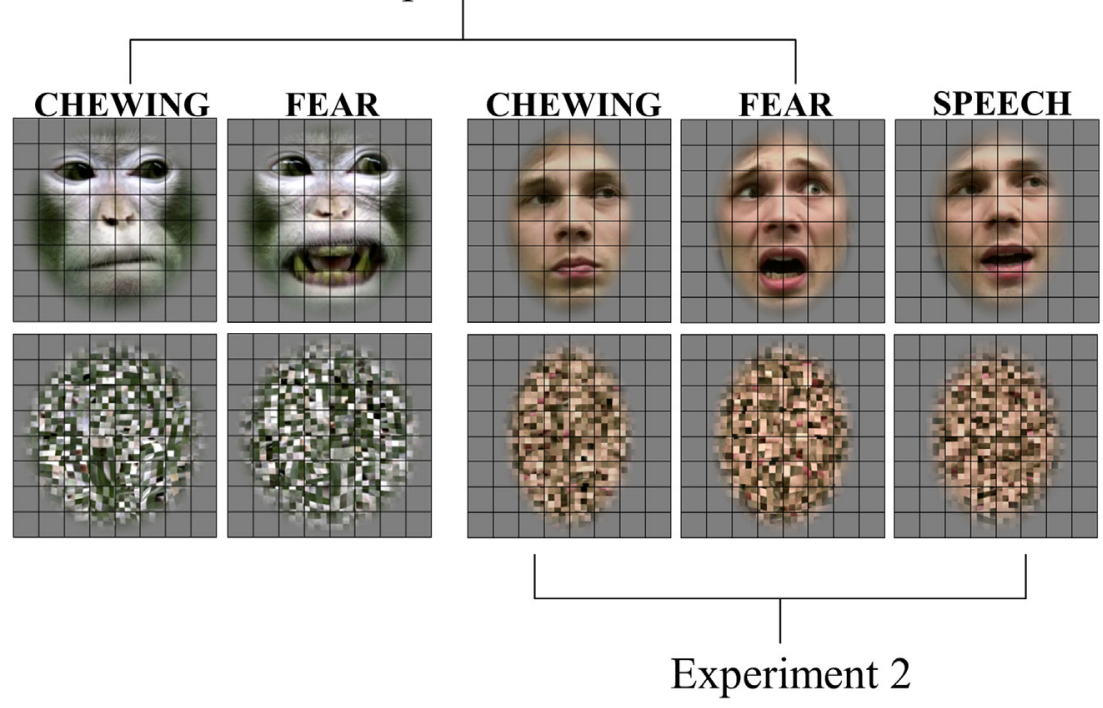

B

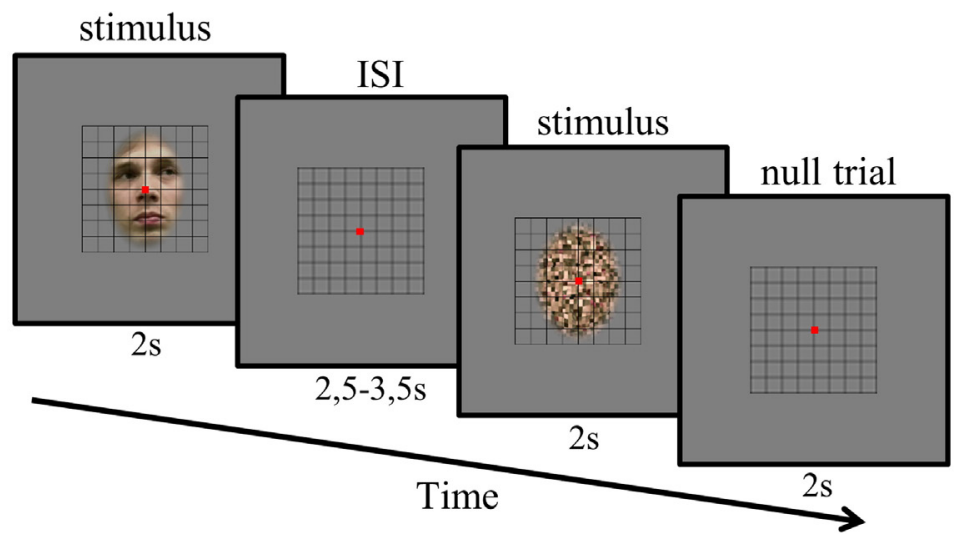

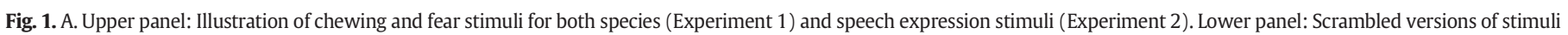
above. B. Illustration of event-related fMRI design for Experiments 1 and 2.

interspersed. Stimuli were presented in ten different orders in Experiment 1; in Experiment 2, stimulus presentation order was different for each run and generated using a genetic algorithm for experimental design (Wager and Nichols, 2003). The size of the stimuli was $7 \times 7^{\circ}$ for both species in Experiment 1 and $5 \times 5^{\circ}$ in Experiment 2. A central fixation point ( $8^{\prime}$ in Experiment 1 and $6^{\prime}$ in Experiment 2 ) was continuously presented and a passive fixation task was performed. Each run lasted $550 \mathrm{~s}$ in Experiment 1 and $360 \mathrm{~s}$ in Experiment 2. To practice, humans viewed a few movie clips prior to Experiment 1 and monkeys were shown static objects unrelated to the experiment. No clips were shown prior to Experiment 2.

After scanning in Experiment 2 we asked subjects to assess the stimuli shown in order to explore if the different expression types were perceived accordingly to the category to which they belonged (see Supplementary materials for methods and results).

\section{Localizer experiment}

We used a block design, sequentially presenting blocks of $16 \mathrm{~s}$ showing 20 static images from one of six categories. Each image was presented for 800 ms during the block. Stimuli were presented exactly as described in Popivanov et al. (2012) for both humans and monkeys, except that a large grid was placed over all stimuli $\left(21.5 \times 21.5^{\circ}\right.$ of visual angle). Presentation of the six categories was preceded and followed by a fixation-only block displaying only the grid on the noise background for $16 \mathrm{~s}$. This was repeated 4 times during each run. The presentation order of all categories was counterbalanced. Three sequences with different presentation orders were used in the same alternating order, both in humans and monkeys. Each run lasted $464 \mathrm{~s}$. A central fixation point $\left(8^{\prime}\right)$ was continuously presented and all subjects performed a passive fixation task.

In all experiments monkeys received liquid rewards for maintaining fixation within a virtual $2 \times 2$ degree window. Fixation performance for every monkey was above $97 \%$ in the analyzed data. Similar eye-tracking data was not available for humans.

\section{fMRI acquisition}

For Experiment 1, humans were scanned on a 3 Tesla Philips Intera scanner using an 8-channel head coil. Experiment 2 and the localizer experiment were performed on a 3 Tesla Philips Achieva scanner using a 32-channel head coil. A standard EPI-sequence (TR $2 \mathrm{~s}$, TE $30 \mathrm{~ms}$, flip angle $90^{\circ}, 40$ slices in Experiment 1, 38 slices in Experiment 2 and localizer scan, $2.75 \times 2.75 \times 3.5 \mathrm{~mm}^{3}$ voxel size) was used. During each scanning session a high-resolution anatomical volume was acquired for each subject using an MPRAGE sequence (TR $9.6 \mathrm{~ms}$, TE $4.6 \mathrm{~ms}$, flip angle $8^{\circ}, 182$ slices, $0.98 \times 0.98 \times 1.20 \mathrm{~mm}^{3}$ voxel size). In Experiment 1 , a total of six runs were obtained in all except four subjects from whom 1 to 2 runs were omitted due to technical problems. In Experiment 2 and in the localizer experiment four runs were obtained in all subjects.

Monkeys were scanned on a 3 Tesla Siemens Trio scanner using an 8-channel monkey coil (TR $2 \mathrm{~s}$, TE $17 \mathrm{~ms}$, flip angle $75^{\circ}, 40$ slices, 
$1.25 \mathrm{~mm}$ isotropic). Before each scanning session a contrast agent (MION 8-11 mg/kg) was injected in the monkey's femoral/saphenous vein. A total of 163,112 and 102 runs from 5,5 and 4 sessions were collected and 144, 103 and 98 runs were analyzed for each monkey respectively. Runs in which monkeys maintained fixation for less than $85 \%$ of the duration of the run (within a $2 \times 2$ degree window) were excluded from the analysis. For the localizer experiment a total of 48 and 59 runs from 2 sessions in two monkeys were analyzed. A high resolution anatomical scan was acquired for each monkey during a separate session under anesthesia using a single radial transmit-receive coil and a MPRAGE sequence (TR $2200 \mathrm{~ms}$, TE 4,05 ms, flip angle 13 $3^{\circ}$, 208 slices, $0.4 \mathrm{~mm}$ isotropic). On the raw EPI images a SENSE image reconstruction was performed to reduce the N/2 ghosting artifacts and correction for higher-order distortions was performed using methods described in Kolster et al. (2009).

\section{fMRI data analysis}

All human data were analyzed using Statistical Parametric Mapping (SPM8; Wellcome Trust Centre for Neuroimaging, London) within Matlab (Mathworks, Inc.). Preprocessing of EPI images included realignment, slice-timing correction (this step was not done for the localizer data), coregistration to the subject's individual high-resolution anatomical image, normalization to the standard template in MNI-space and spatial smoothing using a FWHM of [ 88 8]. After normalization, functional images had dimensions of $53 \times 63 \times 46$ with $3 \mathrm{~mm}$ isotropic voxel size. All stimulus conditions with onsets and duration were entered into a general linear model (GLM); head movement parameters were entered as regressor-of-no-interest for each run. Single subject statistics were modeled by convolving each trial with the canonical hemodynamic response function. For Experiment 1 each half of the runs for every subject was analyzed separately after which two random-effect group analyses were performed on parameter estimates of activity for each contrast across participants using a one-sample t-test. A fixed-effect group analysis was used for Experiment 2 and the localizer experiment.

Motion correction of the monkey data was performed using FS-Fast in Freesurfer (http://surfer.nmr.mgh.harvard.edu/) after skull-stripping of the EPI images. The resulting images were then normalized to the 112RM template (McLaren et al., 2009) based on a non-linear warp field calculated between the T1 image of each monkey and the template using JIP-toolkit (http://www.nmr.mgh.harvard.edu/ jbm/jip/). The spatial smoothing (FWHM $2 \mathrm{~mm}$ ) and further analyses were then all performed in SPM8. In Experiment 1 sessions containing 30 runs or more were equally divided into two separate sessions. Each half of the runs of the resulting 19 sessions was analyzed independently. For GLM analysis each trial was modeled by convolving the MION response function at each trial onset over trial duration. Trials during which monkeys did not fixate were modeled as if fixation occurred. In total this was the case for less than $2.5 \%$ of trials for each monkey. Missed trials did not occur significantly more often in any particular condition (Friedman Test, $\chi^{2}(7)=7.06, p=0.42$ ). Head motion, eye movements and reward schedules were used as regressors-of-no-interest. For Experiment 1 two random effect group analyses were performed on each half of the runs of every session.

Data from the localizer experiment was processed in a similar way except that we used a fixed-effect group analysis. For comparison with BOLD in humans, the sign of the MION signal changes was reversed.

Separating the data of Experiment 1 in two halves allowed us to use one half to define face responsive regions (see definition below) independently from the other half that we used to perform laterality analysis on.

\section{Laterality}

\section{Calculating lateralization}

We used a bootstrapping method within the LI-toolbox for SPM8 (Wilke and Schmithorst, 2006; Wilke and Lidzba, 2007) to explore lateralization within symmetric regions-of-interest for different contrasts in both Experiments 1 and 2. The algorithm was applied on statistical t-maps masked by each ROI (see definition of ROIs below).

The bootstrapping method broadens the data of each ROI by resampling the data under investigation (default: 100 times) and calculating 10,000 lateralization indices (LIs), using voxel values (VV) in Eq. (1), at 20 different statistical thresholds ranging from 0 until the maximum $\mathrm{t}$-value for a specific contrast is reached within the respective ROI. Per threshold a 'trimmed mean' is generated from the middle 50\% of LIs discarding the lower and upper 25\% diminishing the influence of outliers. By plotting these different trimmed means per threshold a curve can be generated for each ROI showing the lateralization at different t-values.

In our analysis the following bootstrapping parameters were applied: sampling size $25 \%$ of input, and $\mathrm{min} / \mathrm{max}$ sample size 5/10,000. No optional steps or exclusive masks were chosen.

$\mathrm{LI}=\frac{\text { VVleft }- \text { VVright }}{\text { VVleft }+ \text { VVright }}$

$\mathrm{LI}_{\mathrm{WM}}=\frac{\sum_{\mathrm{i}=1}^{\mathrm{n}} \mathrm{Wi} * \mathrm{Xi}}{\sum_{\mathrm{i}=1}^{\mathrm{n}} \mathrm{Wi}}$

The trimmed means at each threshold can be used to calculate a weighted mean (LIwm) on the basis of Eq. (2) (adapted from Wilke and Schmithorst (2006)) in which X is a trimmed mean and $W$ is the threshold at which $\mathrm{X}$ was calculated. This yields a single value between -1 and 1 indicating respectively right- or left-sided dominance. We calculated LIwm for several contrasts within different ROIs. In our calculation we only included trimmed means at thresholds where at least $10 \%$ of voxels of the ROI investigated survived in any hemisphere. This was done in order to exclude extreme values at higher thresholds. At such high thresholds LI would be based only on a small amount of voxels that may not be representative for the entire ROI. We therefore also only analyzed ROIs with a voxel count of at least 50 voxels such that by applying this constraint the minimal absolute voxel count in any hemisphere would always be at least 5 , corresponding to the minimum sample size.

An LIwm with a value between -0.25 and +0.25 was defined as 'not lateralized'. Values higher than +0.5 or lower than -0.5 , were considered as strongly lateralized. LIwm values between $+/-0.25$ and $+/-0.5$ were considered as weakly lateralized (Pujol et al., 1999; Lehericy et al., 2000; Kosla et al., 2012).

\section{Defining ROIs}

We first aimed to study laterality in a network responsive to dynamic faces. Secondly we planned to examine if there were differences in laterality between clusters within this network. Finally we looked at laterality within face-selective regions overlapping with the network we defined to see if laterality might be dependent on the definition of the regions.

Dynamic face responsive regions were defined by contrasting dynamic human and monkey faces versus their scrambled versions within one half of the data derived from Experiment 1 (see above). T-score maps were thresholded at an uncorrected level $\mathrm{p}<0.00001$. A mask was created from the resulting significance map. The original and flipped masks were then merged to generate an unbiased bihemispheric symmetrical mask used for the laterality analysis. Clusters within this mask were also used as separate masks. To define faceselective masks, we used the face localizer data by contrasting human and monkey faces versus objects and thresholded maps at voxel-level $\mathrm{p}<0.001$ FWE corrected for multiple comparisons. A bi-hemispheric mask was created in a similar manner and regions overlapping with the face responsive mask were further used. 
After calculating laterality for face responsive contrasts we planned to further investigate the effect of different expression types in those regions exhibiting lateralization for faces.

Further for all human subjects we calculated individual LIwm values for the ROIs and contrasts under investigation at group level in order to search for statistical differences in lateralization for those different ROIs and contrasts.

\section{Results}

Regions of interest

Face responsive regions

In both humans and monkeys, we found several clusters containing voxels that were responsive to faces in either left and/or right occipital and temporal cortexes. For humans these were the ventro-lateral occipito-temporal cortex (vlOT) and the posterior superior temporal cortex (pSTS). Because these two clusters encompassed more than one of previously reported face responsive regions (Kanwisher and Barton, 2011; Pitcher et al., 2011), we additionally made further subdivisions by separating the pSTS cluster in two parts along the $y$-axis by cleaving the cluster at the center between the most anterior and posterior coordinates, resulting in an anterior (a-pSTS) and a posterior ( $\mathrm{p}$-pSTS) subdivision of pSTS. A similar differentiation was made along the $\mathrm{z}$-axis for the vlOT cluster, resulting in a lateral occipito-temporal (IOT) and a ventral occipito-temporal cluster (vOT) (Figs. 2A-C). Another cluster including amygdala (AMG) came short to matching our required minimum of a voxel count of 50 (AMG: 47 voxels) but for completeness we chose to include it in the group analyses (Fig. S1).

Face-responsive clusters in monkey consisted of an occipital cluster in V1, a large occipito-temporal cluster extending in the superior temporal sulcus and a region located in the anterior part of the inferotemporal cortex (aIT) (Fig. 2D). As hemispherical asymmetries are more likely to arise at higher levels of visual processing where retinotopic organization breaks down (Corballis, 2003), we differentiated early visual cortical regions from regions further down the ventral visual stream by masking the occipito-temporal cluster and the cluster in V1 with bilaterally merged probabilistic retinotopic maps of areas V1, V2, V3, V4 and V4A (Fig. 2E). These maps were obtained from three macaque monkeys (Janssens et al., 2014), two of which participated in Experiment 1 . This allowed us to separate the occipito-temporal cluster into a more anterior part including the middle part of the STS (thus referred to as mid-temporal cluster), and create a more posterior part containing activations in early visual area V1, V3, V4 and V4A (thus referred to as V1-V4A cluster) (Fig. 2F).

\section{Face selective regions}

In humans, we found a cluster in ventral temporal cortex (FFA) and one in superior posterior temporal cortex (pSTS) which in analogy to the overlapping face responsive region we subdivided into an anterior (a-pSTS) and a posterior part (p-pSTS) (Figs. 3A and B).

In monkeys we encountered two regions in temporal cortex: an anterior temporal one (aIT) and a mid-temporal one (Fig. 3C).

\section{Lateralization for dynamic face processing in humans}

\section{Face-responsive network (Fig. 4 upper panel)}

We first explored lateralization for dynamic face processing within the entire bi-hemispheric volume of all face responsive voxels. Contrasting human and monkey faces versus their scrambled versions did not reveal lateralization. We found a weak right hemispheric lateralization when contrasting human faces versus scrambled versions but no lateralization for monkey faces versus scrambled versions.
A

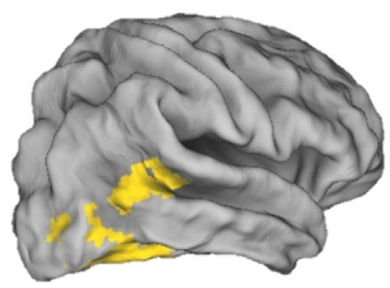

$\mathrm{D}$

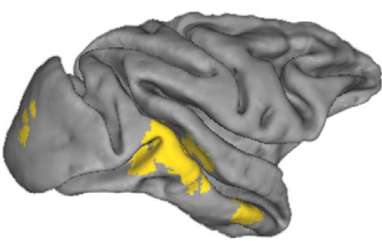

B

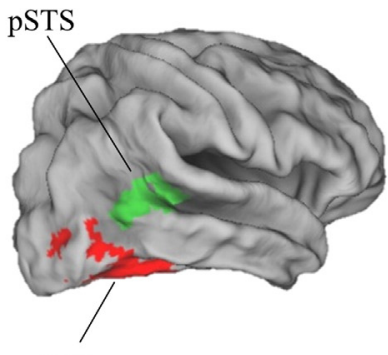

vlOT
$\mathrm{C}$

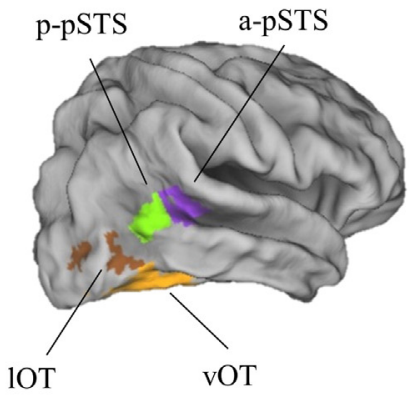

F

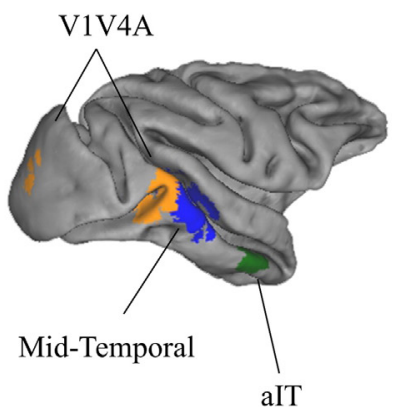

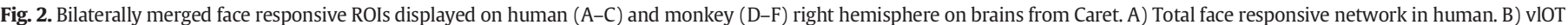

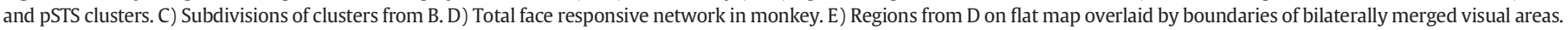

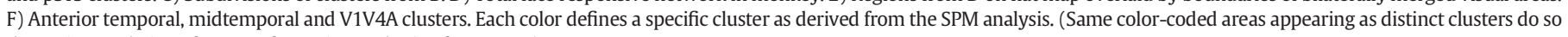
due to interpolation after transformation on brains from Caret). 
Face-responsive clusters (Fig. 4)

The vlOT region showed no lateralization for any face responsive contrast. Also in the two subdivisions of this cluster, we did not find any evidence for lateralization for dynamic faces.

In pSTS, on the other hand, we found strong right-sided lateralization when contrasting all faces versus scrambled versions and only monkey faces versus scrambled versions. For human faces versus scrambled versions we found weak right-sided lateralization. Along the posterior to anterior axis within this cluster lateralization increased from weak towards strong for all face responsive contrasts.

Finally, in amygdala no lateralization was found for the three face responsive contrasts.

\section{Face-selective areas (Fig. 5 upper panels)}

FFA showed no lateralization for the face responsive contrasts. The pSTS, however, showed a weak right-sided lateralization for these same contrasts. Again here, the highest degree of lateralization was found within the anterior subdivision of pSTS with strong right-sided lateralization, except for human faces versus scrambled versions where the LIwm value only just did not reach the criterion. Weak lateralization was found in p-pSTS for human faces and for monkey faces contrasted with their scrambled versions.

\section{Inter-individual variance for dynamic face processing}

After exploring lateralization at group activation level for dynamic face processing, we examined this further at the individual level using exactly the same ROIs. Our special interest pertained to differences in lateralization between ventral lateral occipito-temporal areas and pSTS as well as differences between p-pSTS and a-pSTS. Fig. 6 illustrates the variability of LIwms between subjects per contrast and per ROI. Parametric tests were used to describe the variance as no violation of normality assumption was revealed after testing for normality using Shapiro-Wilk test (alpha set at 0.01). A one sample t-test (uncorrected) on the individual LIwms for each ROI per contrast showed that only the pSTS had values that significantly differed from zero $(t(19)=-3.369$, $\mathrm{p}=0.003 ; \mathrm{t}(19)=-3.529, \mathrm{p}=0.002 ; \mathrm{t}(19)=-2.403, \mathrm{p}=0.027$ for the face responsive ROI and $\mathrm{t}(19)=-2.957, \mathrm{p}=0.008 ; \mathrm{t}(19)=$ $-3.427, \mathrm{p}=0.003 ; \mathrm{t}(19)=-2.092, \mathrm{p}=0.05$ for the face selective ROI for the contrasts of all faces, human faces only and monkey faces only versus scrambled versions respectively), with the strongest effect in its anterior subdivision $(\mathrm{t}(19)=-4.217, \mathrm{p}<0.0005 ; \mathrm{t}(19)=$ $-3.230, \mathrm{p}=0.004 ; \mathrm{t}(19)=-4.130, \mathrm{p}=0.001$ for the face responsive ROI and $\mathrm{t}(19)=-3.628, \mathrm{p}=0.002 ; \mathrm{t}(19)=-3.399$, $\mathrm{p}=0.003 ; \mathrm{t}(19)=-3.629, \mathrm{p}=0.002$ for the face selective ROI for the same contrasts).

We then examined if lateralization in pSTS differed significantly from other areas and whether this was contrast-dependent, using a repeated measures ANOVA with contrast and region as within subject factors. We found that mean LIwm differed significantly between regions $\left(F(2.637,50.096)=5.288, p<.005, \eta_{p}^{2}=.0218\right.$; with Greenhouse-Geisser correction) but not between contrasts nor did we find an interaction between region and contrast. Post-hoc pairwise comparisons (uncorrected) between face responsive ROIs revealed a significant difference between vlOT and pSTS $(\mathrm{p}=0.027)$ as well as between vlOT and a-pSTS ( $p=0.007)$; between vOT and a-pSTS ( $p=0.019)$; between IOT and pSTS $(\mathrm{p}=0.002)$ and its posterior $(\mathrm{p}=0.016)$ and anterior subclusters $(p=0.002)$. Further we found a significant difference between $\mathrm{p}$-pSTS and a-pSTS $(\mathrm{p}=0.044)$. Between face selective ROIs there was a significant difference between FFA and a-pSTS $(\mathrm{p}=0.02)$ (Fig. 6).

\section{Effect of type of expression (Fig. 7)}

Finally in a separate experiment in humans (Experiment 2), we examined whether lateralization we found in face responsive pSTS might be influenced by the type of facial expression perceived. As expected, chewing and fearful faces compared to their scrambled versions showed a strong lateralization to the right within pSTS and apSTS. In p-pSTS we found weak lateralization for fear and strong for chewing. Speech stimuli, on the other hand, showed no lateralization when contrasted to their scrambled versions in STS and in a-pSTS while also a weak right-sided lateralization was found in p-pSTS. When we contrasted speech directly with chewing or fearful faces (controlled for scrambled versions), we found strong left-sided lateralization in pSTS, and in a-pSTS. On the other hand we found only weak lateralization for speech versus chewing and none for speech versus fear in p-pSTS. The emotion specific contrast of fear versus chewing was also examined within these ROIs but as voxel count dropped below the required minimum (10\% of ROI) in both hemispheres beneath a t-value of 2.0, it is hard to draw any conclusions from resulting LI values. We therefore chose not to report these results.

Exploring laterality for the same contrasts as above within the faceselective pSTS and its subregions yielded similar results (Fig. S2).

\section{Inter-individual variance for different types of expressions}

Analogous to Experiment 1 we examined lateralization at the individual level in Experiment 2. Individual LIwm values for the contrasts chewing, fear and speech versus their respective scrambled versions and speech versus chewing and fear (controlled for scrambled versions) were calculated in pSTS as well as in its subclusters for face responsive and face selective clusters (Figs. 8 and S3). We searched for significant differences in lateralization between contrasts as well as differences between ROIs. As normality of samples could not be assumed in 9 of 30 samples examined non-parametric tests were used for further comparisons, Friedman Test (uncorrected). In total there were 8 missing data points across 2 subjects due to voxel count below the required minimum in any hemisphere at the start of calculation.

For face responsive regions we found significant differences in lateralization between regions for the contrasts fear versus scrambled versions $\left(\chi^{2}(2)=8.0, \mathrm{p}=0.018\right)$ and speech versus fear $\left(\chi^{2}(2)=\right.$ $9.6, \mathrm{p}=0.008)$. Post hoc testing revealed that there was a stronger right-sided lateralization in a-pSTS than in p-pSTS for fear versus
A

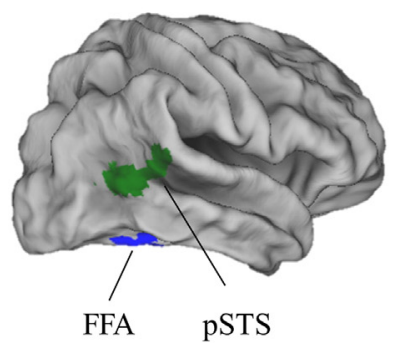

B

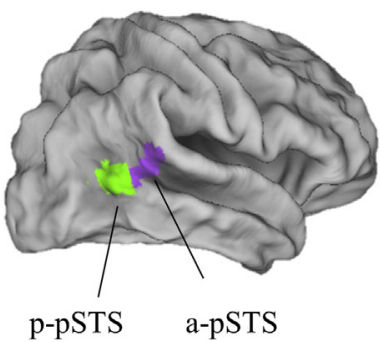

$\mathrm{C}$

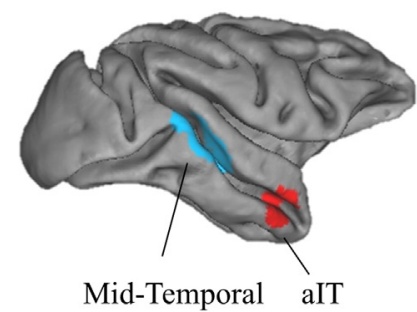

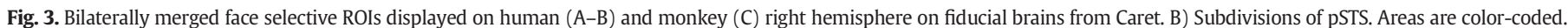



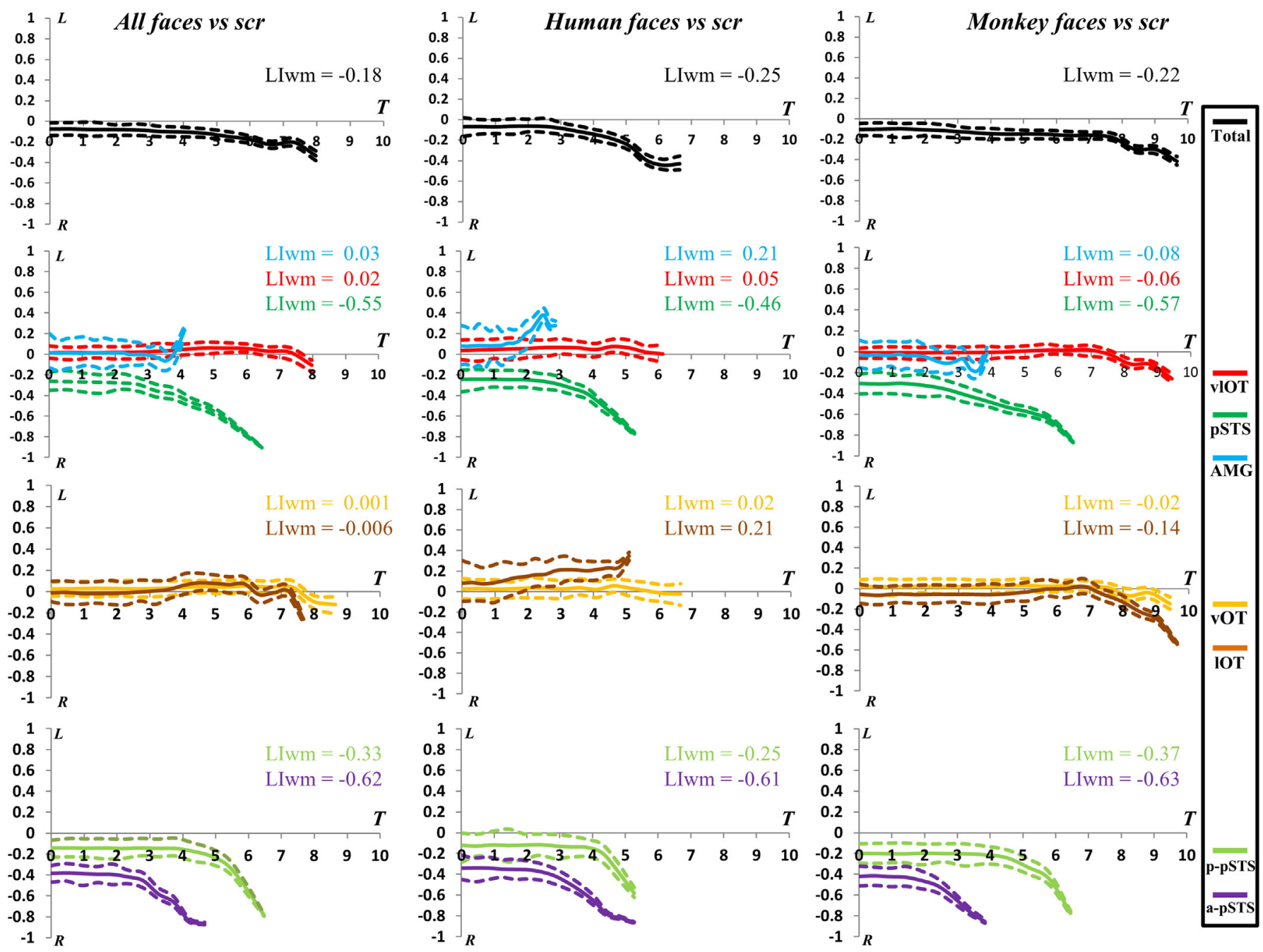

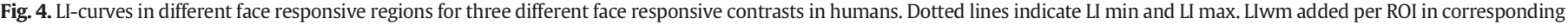
color. Y-axis: lateralization index; X-axis: thresholded t-value. $\mathrm{L}=$ Left-sided, $\mathrm{R}=$ right-sided. Scr $=$ Scrambled versions.

scrambled versions $\left(\chi^{2}(1)=5.4, \mathrm{p}=0.02\right)$ as well as a stronger leftsided lateralization in pSTS than in its posterior subcluster for speech versus fear $\left(\chi^{2}(1)=10.0, p=0.002\right)$. We also observed a trend towards a significant difference between p-pSTS and a-pSTS for this contrast $\left(\chi^{2}(1)=3.6, \mathrm{p}=0.058\right)$.

We found significant differences in lateralization for all face responsive regions across the five contrasts we examined (pSTS: $\chi^{2}(4)=28.2$, $\mathrm{p}<0.001$; p-pSTS: $\chi^{2}(4)=16.8, \mathrm{p}=0.002$; a-pSTS: $\chi^{2}(4)=22.5, \mathrm{p}<$ $0.001)$. Pairwise comparisons in a post hoc test revealed no differences in any region between chewing and fear versus their scrambled versions. Within pSTS we found significantly less negative values for speech versus scrambled versions than for chewing or fear $\left(\chi^{2}(1)=\right.$ $6.4, \mathrm{p}=0.011)$. Between speech and fear versus their scrambled versions there was also a significant difference within a-pSTS $\left(\chi^{2}(1)=\right.$ $9.0, p=0.003)$. Between chewing versus scrambled versions and speech versus chewing we observed a significant shift to the left within pSTS but not within its subclusters separately $\left(\chi^{2}(1)=5.4, \mathrm{p}=0.02\right)$. Between fear versus scrambled versions and speech versus fear we also found a significant shift in lateralization to the left within pSTS as well as in p-pSTS and a-pSTS $\left(\chi^{2}(1)=9.0, p=0.003 ; \chi^{2}(1)=10.0\right.$, $\mathrm{p}=0.002$ and $\chi^{2}(1)=6.4, \mathrm{p}=0.011$ respectively). We did not find any significant difference in any ROI between speech versus chewing and speech versus fear.

A similar analysis for face selective pSTS and its clusters yielded similar results (Fig. S3).
Lateralization in monkeys

Face-responsive network (Fig. 9, upper panel)

We did not find lateralization within the entire face-responsive network in monkeys for any of the face responsive contrasts.

Face-responsive clusters (Fig. 9, lower panel)

When studying lateralization within individual clusters, we found weak right-sided lateralization in the aIT but only for the contrast human faces versus scrambled versions, which was mainly driven by voxels at higher thresholds (t-values $>4$, see Fig. 9). For the other face responsive contrasts we did not find any lateralization. No lateralization was found in the other clusters.

\section{Face-selective areas (Fig. 5, lower panel)}

As in humans, we also studied lateralization in face selective regions. Except for a weak left-sided lateralization in aIT for human faces versus scrambled versions we did not find any lateralization for other contrasts or within the midtemporal cluster.

\section{Effect of type of expression}

As we did for humans in Experiment 2 we further studied the effect of different expression types for monkey within Experiment 1 (Fig. 10). We investigated if the weak lateralization we found in aIT might be 
All faces vs scr
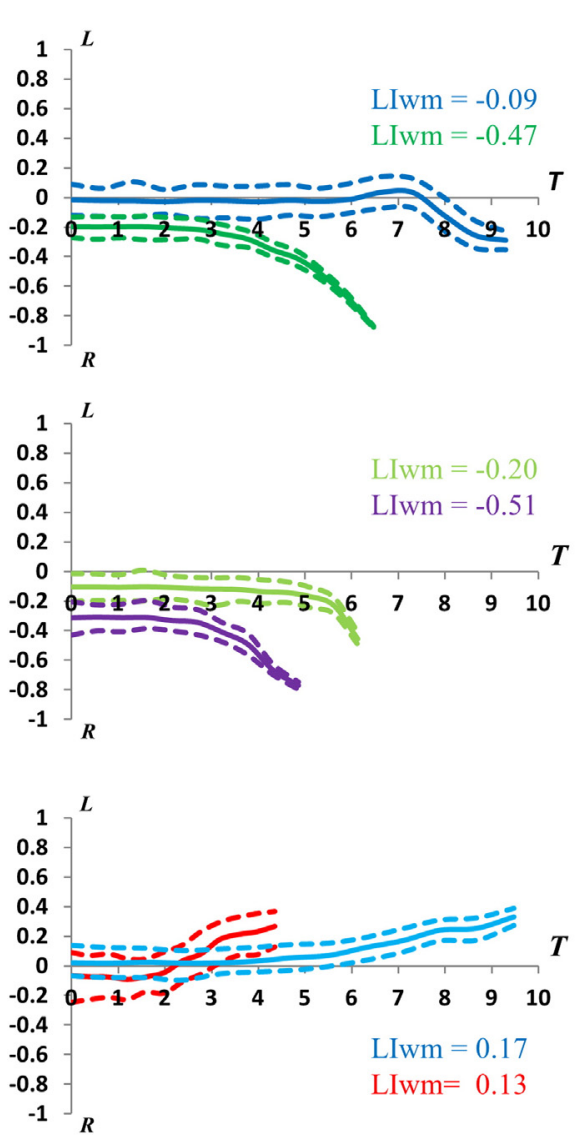

Human faces vs scr

HUMAN
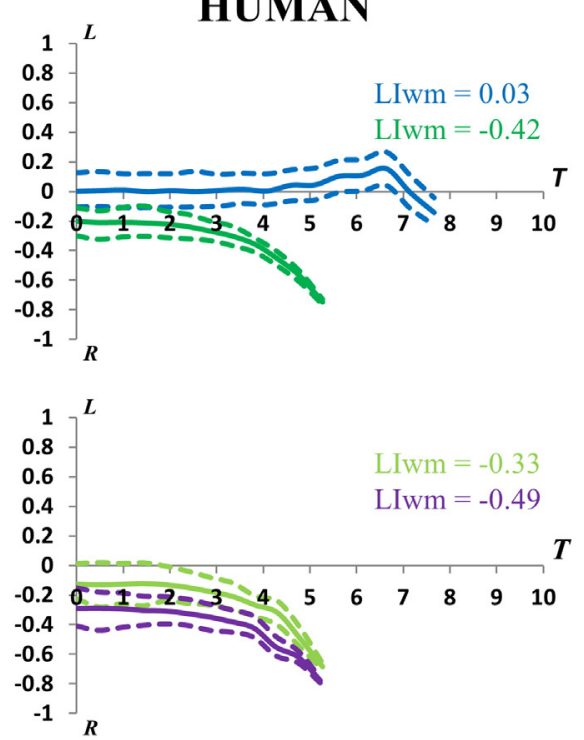

MONKEY

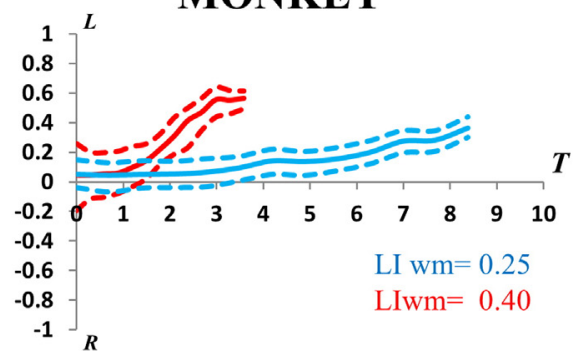

Monkey faces vs scr
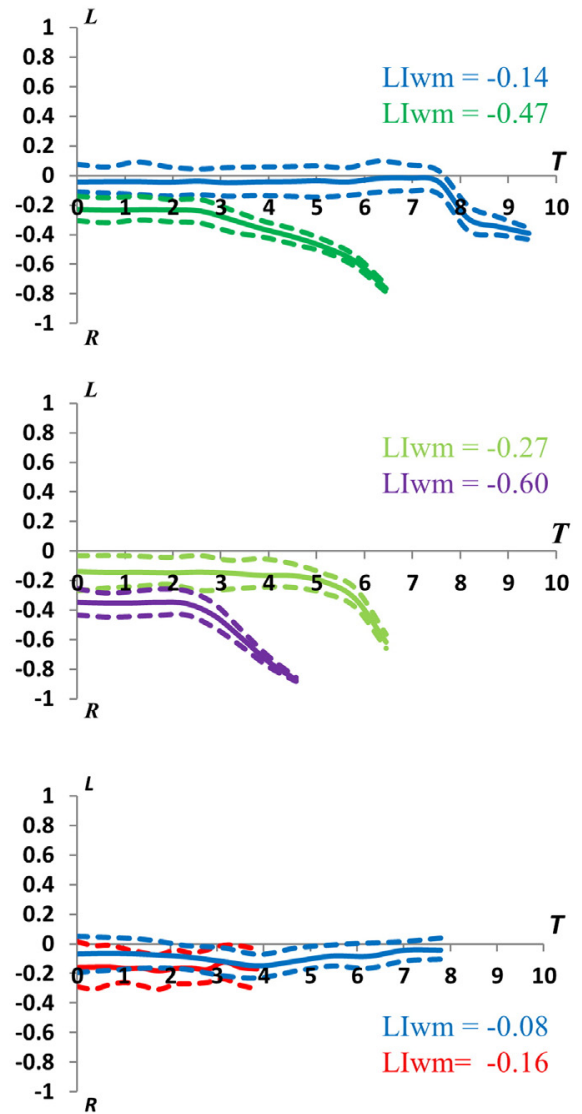

FFA

pSTS

p-pSTS

a-pSTS

aIT

Mid-Temporal

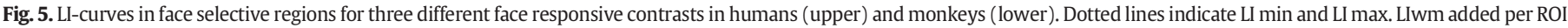
in corresponding color. Y-axis: lateralization index; X-axis: thresholded t-value. $\mathrm{L}=$ Left-sided, $\mathrm{R}=\mathrm{Right}$-sided. Scr $=\mathrm{Scrambled}$ versions.

dependent on expression type. We did not find lateralization nor for chewing faces nor for fearful faces versus scrambled versions in face responsive aIT. In face selective aIT we found weak left-sided lateralization for chewing faces but not for fearful faces versus scrambled versions.

For the same reason as in humans lateralization for fear versus chewing is not reported here (voxel count dropped below the required minimum in both hemispheres at $\mathrm{t}$-value $<2.5$ ).

\section{Discussion}

We found consistent lateralization to the right for chewing and emotional dynamic faces in human pSTS, most pronounced in its anterior section. This finding was independent of how exactly the regions of interest were defined. When we used the same stimuli in monkeys, no consistent pattern of lateralization emerged. In humans an additional visual speech stimulus did not reveal lateralization when compared to scrambled controls while a shift towards the left was observed when compared to other facial expressions within the same pSTS ROI.

In their seminal paper, Hamilton and Vermeire (1988) argued that the right-hemispheric bias for face processing was independent of handedness or language development as other studies in monkeys also observed lateralization (Jason et al., 1984). Along this line of reasoning, different theoretical models have been proposed to account for the right hemispheric bias for facial stimuli. These models argue that the right hemisphere ( $\mathrm{RH})$ employs different object processing strategies compared to the left hemisphere (LH), such as configural versus analytic, parallel versus serial, specificity for low versus high spatial frequency stimuli, specific-exemplar versus abstract-category or spatial metrics versus associative co-occurrence (for review see Dien (2009)). According to these models, the RH processing strategies that are assumed to be ubiquitous in primates predispose to lateralized processing of faces. The alternative view is that the right-hemispheric bias for faces evolved as a cost associated with left hemispheric specialization in processes that support language functions (Corballis et al., 2000). Classically, this model is referred to as the verbal versus visuospatial model because the original descriptions capitalized on the cooptation of cortical tissue in the LH previously dedicated to visuospatial processing by linguistic processes (Milner, 1971; Corballis et al., 2000). Although both views predict laterality for faces in humans (Fitch et al., 2010), the fundamental premise that differs between these hypotheses is that according to the former theory a similar degree of laterality is expected when the same non-linguistic face stimuli are presented to monkeys or humans. Our data argue against this point of view: in contrast to the $\mathrm{RH}$ lateralization in humans, no consistent lateralization for dynamic faces was observed in monkeys. Moreover, lateralization for non-linguistic facial stimuli in humans was mainly confined to the right pSTS area of the face recognition system. The left-hemispheric mirror region of this area is considered as a crucial component of the language network, more particularly it is involved in mapping input from different modalities on lexical representations (Calvert et al., 2000). The relative higher activity in the left pSTS for facial speech expressions compared to other expressions, which we 


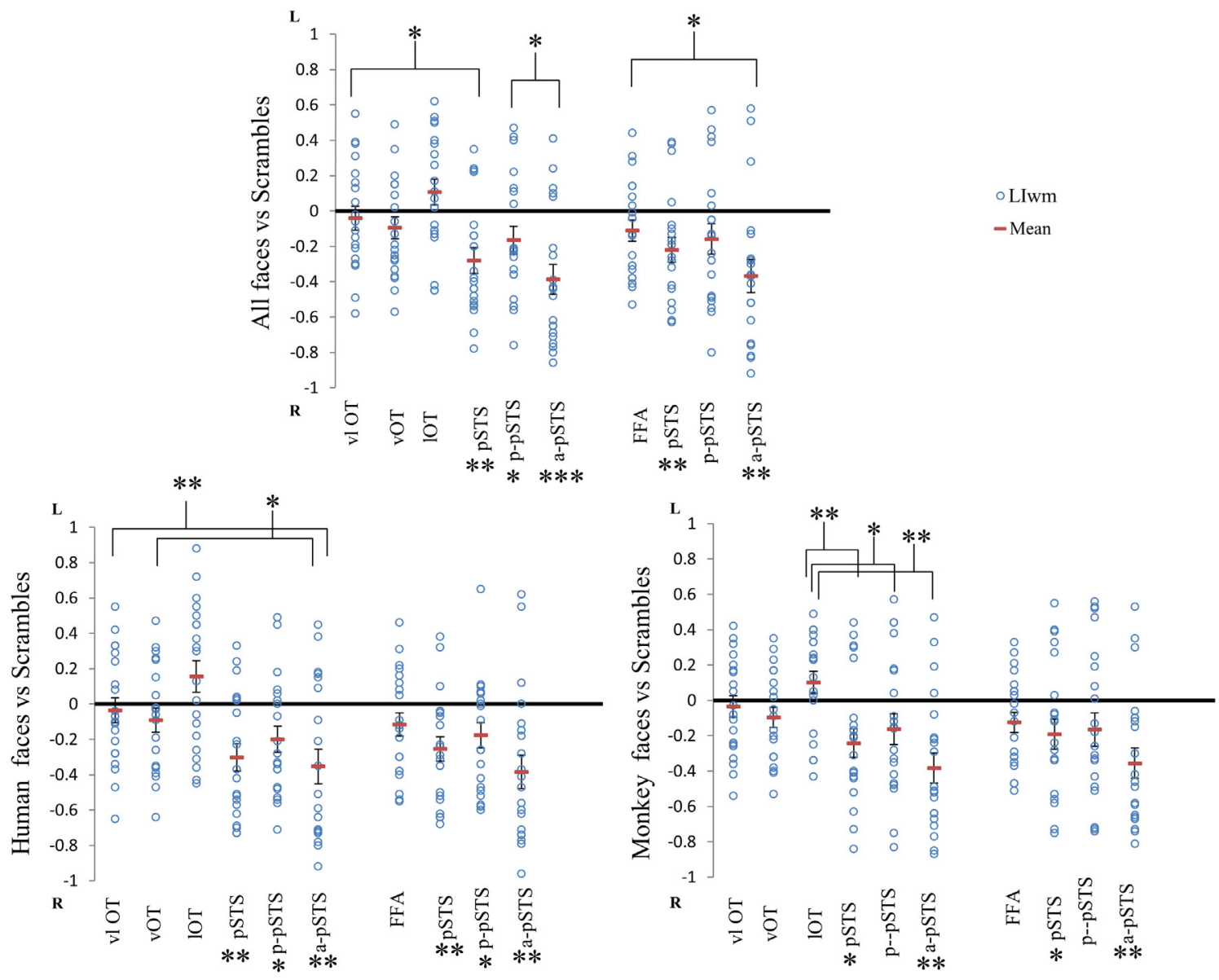

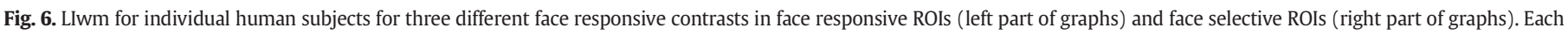

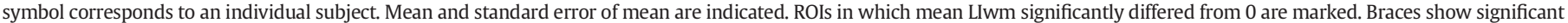
differences in mean Llwm between ROIs as described in text. ${ }^{*} \mathrm{p}<0.05,{ }^{* *} \mathrm{p}<0.01,{ }^{* * *} \mathrm{p}<0.001$. $\mathrm{L}=$ Left-sided, $\mathrm{R}=$ right-sided.

confirmed in the present study, has been proposed to reflect this cognitive process (Calvert et al., 1997; Campbell et al., 2001; MacSweeney et al., 2002; Hall et al., 2005; Bernstein et al., 2011). In addition to previous studies that used different control conditions (Calvert et al., 1997; Campbell et al., 2001; Paulesu et al., 2003), we showed that there is little lateralization for facial speech perception compared to scrambled versions in superior temporal cortex, in contrast to the clear lateralization that we observed for other expressions. The absence of lateralization for facial speech perception compared to scrambled versions could be the result of concurrent activation of the right and left pSTS, where the former region is thought of as a common processing site for dynamic facial representations (Campbell et al., 2001) and the latter one is thought to contain an area for prelexical visual cue integration (Bernstein et al., 2011). In other words, speech related expressions probably elicit activation in both left and right STS, associated with language and face processing respectively, resulting in bilateral activation and no lateralization. This is corroborated by that fact that once the face processing component is controlled for, i.e. the contrast between speech and chewing or fear, lateralization to the left becomes evident. Anyway, the comparison between monkey and human data together with the expression-dependent effect in human STS suggests that language development might have played an essential role in lateralization for dynamic face processing.

Surprisingly, lateralization for dynamic faces was present in human STS, but not in face-responsive ventral occipito-temporal cortex nor in face-selective area FFA. This finding is at odds with the majority of static face studies reporting stronger activations in the right compared to the left FFA (Dien, 2009). It should be noted, however, that only a limited number of studies performed formal statistical testing on laterality, some showing a right-sided lateralization (e.g. Haxby et al., 1999) while others did not (e.g. Ishai et al., 2002). It is possible that the higher amount of visual information that needs to be processed by fusiform cortex for dynamic versus static faces (LaBar et al., 2003; Sato et al., 2004) is associated with bilaterally enhanced neural activity and less lateralization. Human STS on the other hand is a multimodal associative brain region which depends less on visual input. According to the model proposed by Haxby and Gobbini, different aspects of visual information reach STS and fusiform cortex independently. Recent studies confirm that the connectivity patterns of both areas differ substantially without direct connections between these areas. Whereas FFA seems to be part of an extrastriate ventral pathway, STS is primarily connected with fronto-parietal cortex probably mediating higher order social cognitive processes (Gschwind et al., 2012). The lack of connectivity between STS and FFA observed in the study of Gschwind et al. (2012) is different from the strong connectivity that has been reported between the face patches in monkeys (Moeller et al., 2008). Moreover, recently, it has been proposed that specialization for dynamic facial expressions in human STS is unmatched by monkey face areas (Zhu et al., 2013; Polosecki et al., 2013). The human-specific laterality pattern that we observed provides an additional argument in favor of the idea that human STS developed properties that are not present in monkeys.

Although most face studies support right-hemispheric specialization, there are inconsistent reports with occasional LH biases depending on task parameters (Dien, 2009). One of the parameters that may contribute to variations in lateralization strength and direction is the 


\section{CHEWING VS SCR}

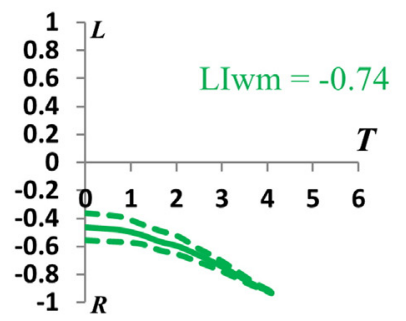

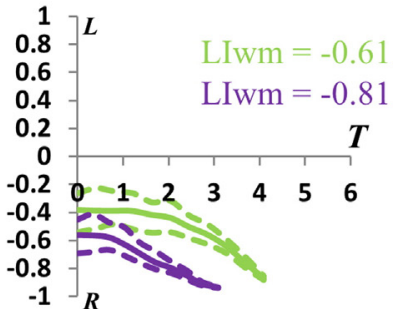

FEAR VS SCR

\section{SPEECH VS SCR}

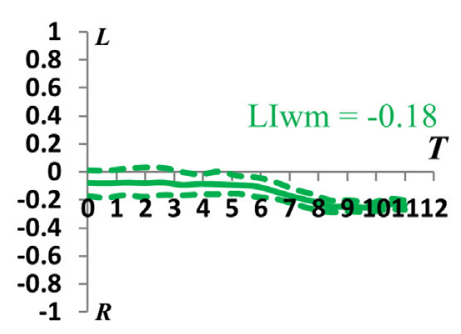

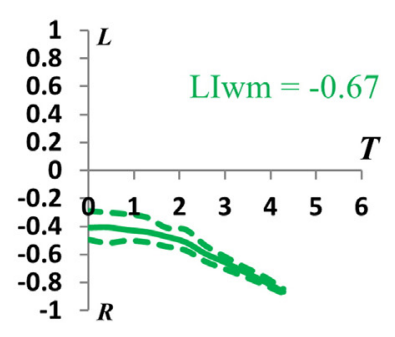

SPEECH VS CHEWING

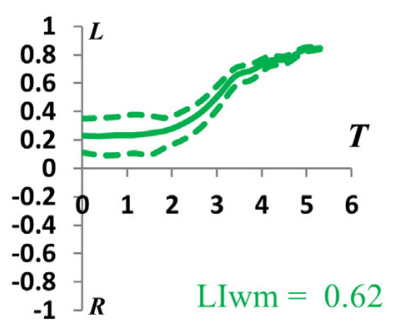

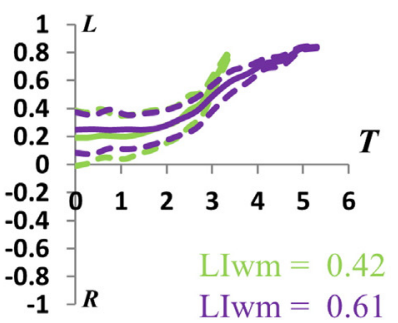

pSTS $-\mathrm{p}-\mathrm{pSTS}$

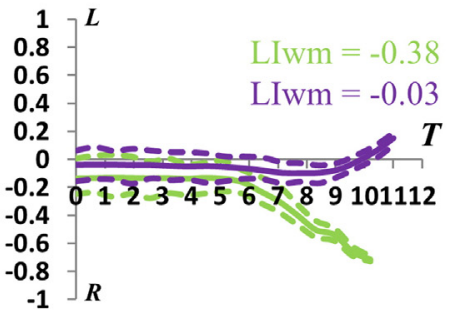

SPEECH VS FEAR
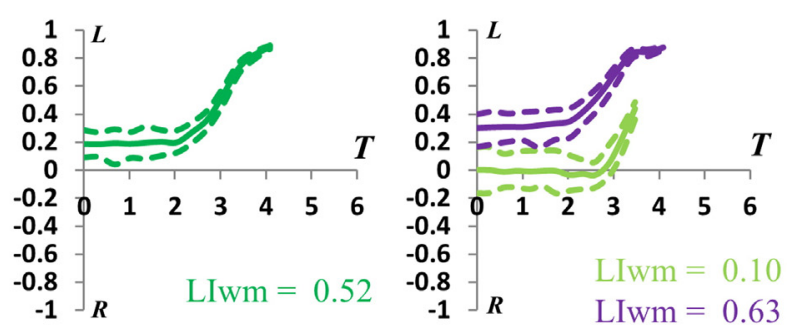

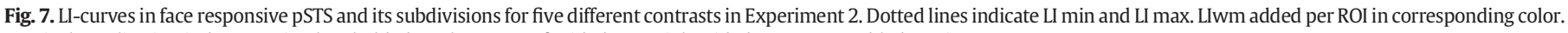
Y-axis: lateralization index; X-axis: thresholded t-value. $\mathrm{L}=$ Left-sided, $\mathrm{R}=$ right-sided. $\mathrm{Scr}=\mathrm{Scrambled}$ versions.

chosen contrast, which varies between objects, scrambles, fixation and static controls for dynamic stimuli. It has been proposed that in studies with static faces using objects as a control condition favors righthemispheric lateralization for faces by subtracting out more general object recognition processes (Kanwisher et al., 1997; Dien, 2009). More recently Meng et al. showed that activation patterns in the left FFA correlate with face-semblance while in the right FFA this correlates with categorical face/non-face judgments, which would favor a bilateral activation pattern in this region in our design (Meng et al., 2012). However, contrasting moving faces with moving objects in the study of Polosecki et al. (2013) reliably revealed left STS in humans. The contrast between moving and static faces on the other hand corroborates our findings (Pitcher et al., 2011). The primary reason to choose scrambled versions as controls in our study was to control for differences in local motion, which is inevitable when using moving objects or static faces. Given the motion sensitivity of STS, we considered local motion as an important potential confound. Our factorial design allowed us to rule out - as much as possible - that differences in local motion would account for the differences between expressions.

The suggestion that the processing of emotional cues such as facial expressions may be more lateralized to the right has a long history, mainly rooted in clinical observations of patients with brain lesions.
Evidence from functional imaging studies remains limited (Fusar-Poli et al., 2009) with some exceptions that included rigorous testing (Noesselt et al., 2005). Our study shows that the notion of disproportionate lateralized processing of emotional expressions compared to other expressions does not apply to dynamic facial stimuli, at least not in the areas that we examined. We also did not observe differences in laterality effects between human faces and monkey faces, pleading against species-specific lateralized processing strategies of non-verbal expressions. This finding fits in with the similar activation found for faces across species in humans and the wide range of face-like stimuli able to be detected by face patches in monkeys (Tong et al., 2000; Tsao et al., 2003).

Although we found a right-sided lateralization within human posterior superior temporal cortex, its anterior part showed stronger lateralization than the posterior subdivision for non-verbal expressions. The lateralization pattern in p-pSTS was also less affected by speech as facial expression than in a-pSTS. Strikingly a similar disparity concerning dynamic face selectivity in these regions has been demonstrated with the strongest selectivity in the anterior STS (Pitcher et al., 2011). Thus there is a convergence between selectivity and lateralization for dynamic faces within pSTS with laterality decreasing when a language-related stimulus was introduced. 


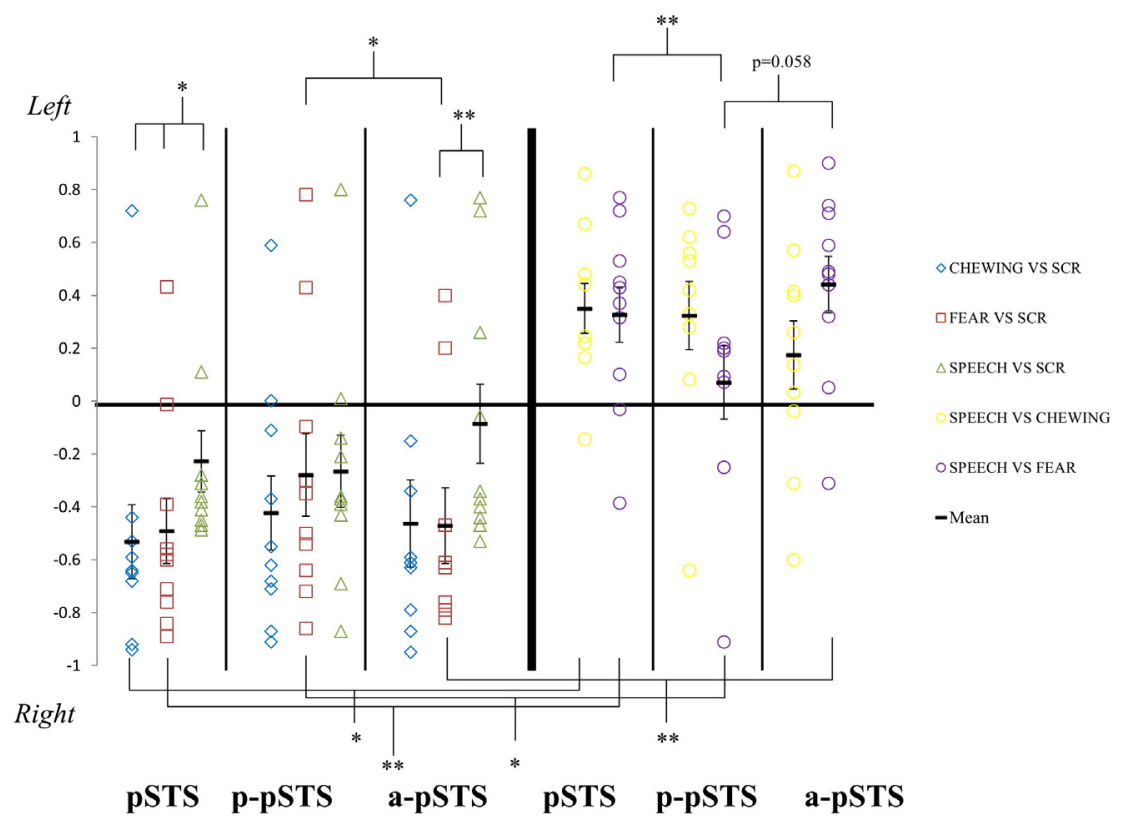

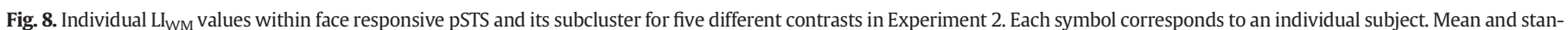

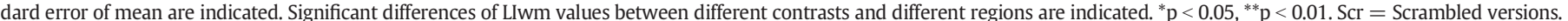

It should also be noticed that we found important inter-individual differences in the magnitude of lateralization in pSTS and even lateralization to the left (positive LIwms) in 3 to 6 subjects depending on the contrast examined. We acknowledge that lateralization for language processing is more consistent across right-handed individuals (92.5 to 100\%) (Springer et al., 1999; Knecht et al., 2000) compared to the lateralization for dynamic face processing in pSTS ( 70 to $85 \%$ depending on contrast) in our study. Similar variability (78\% right-hemispheric lateralization) has been described by Bukowski et al. for static faces in STS (Bukowski et al., 2013).
A possible explanation is that non-speech dynamic facial expressions including movements of the mouth elicit semantic associations in some individuals, associated with activation of components of a left hemispheric semantic network including the left pSTS (Vandenbulcke et al., 2007). Relating to this, preliminary results reported in Bernstein et al. (2011) show that activation for non-speech dynamic facial stimuli in a region crucial for visual speech processing in the left pSTS shows a marginally negative correlation with lipreading performance (Bernstein et al., 2011). Another explanation could be that language development contributed critically but not exclusively to lateralization for dynamic
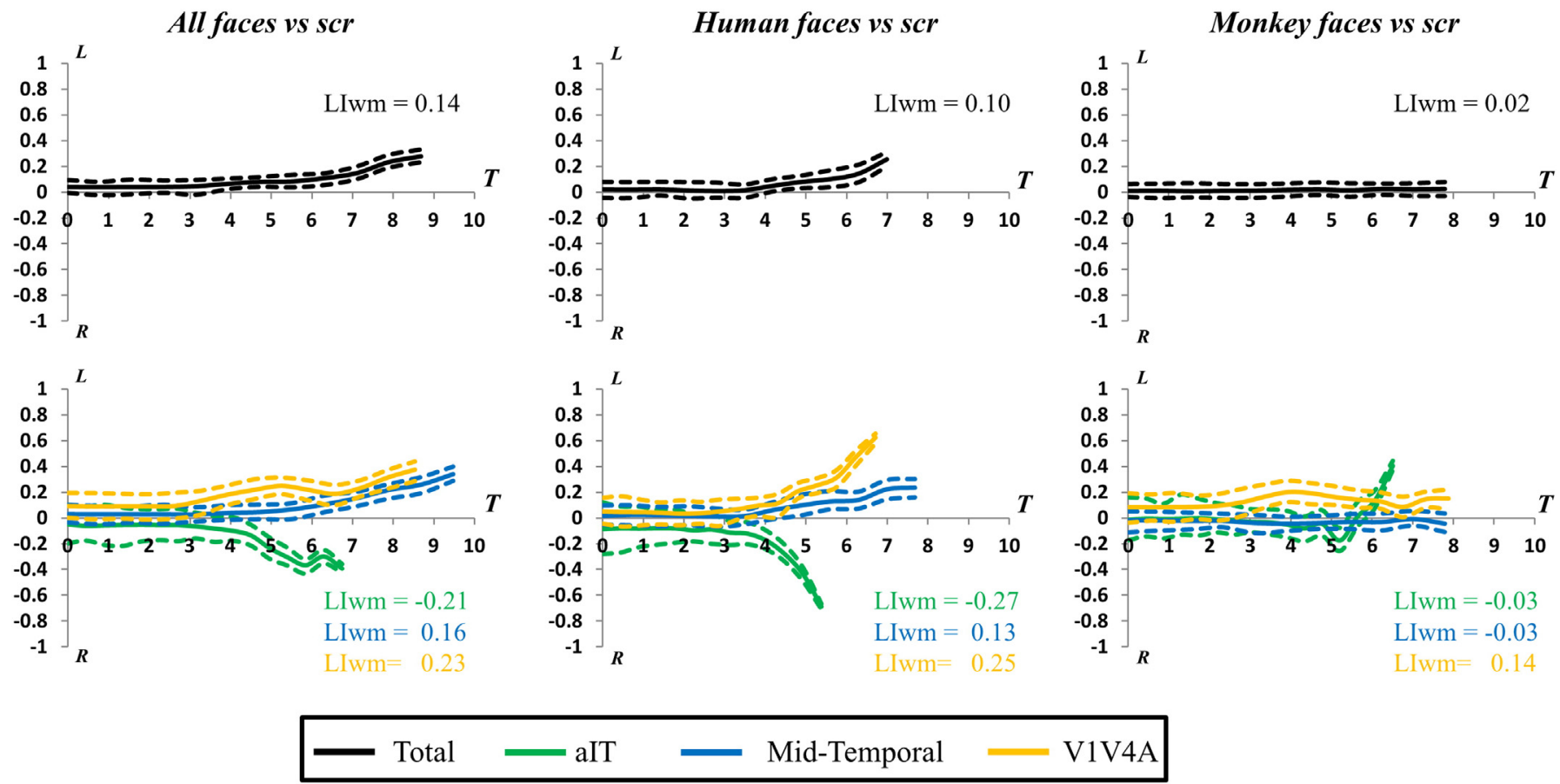

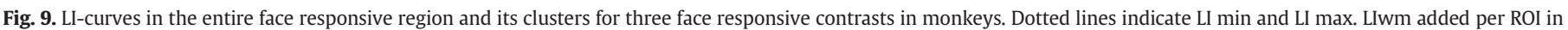
corresponding color. Y-axis: lateralization index; X-axis: thresholded t-value. $\mathrm{L}=$ Left-sided, $\mathrm{R}=$ right-sided. Scr $=\mathrm{Scrambled}$ versions. 
Chewing faces vs scr

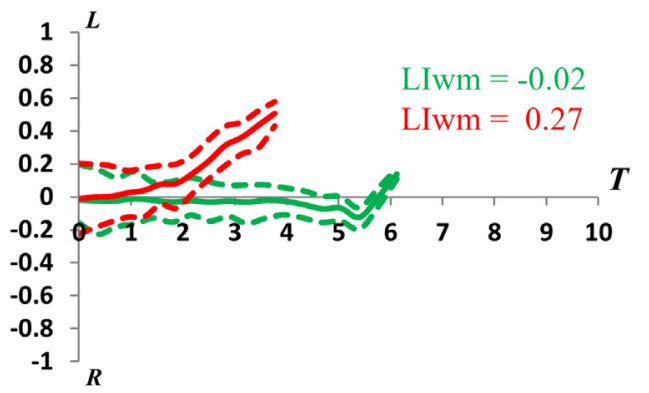

Fearful faces vs scr

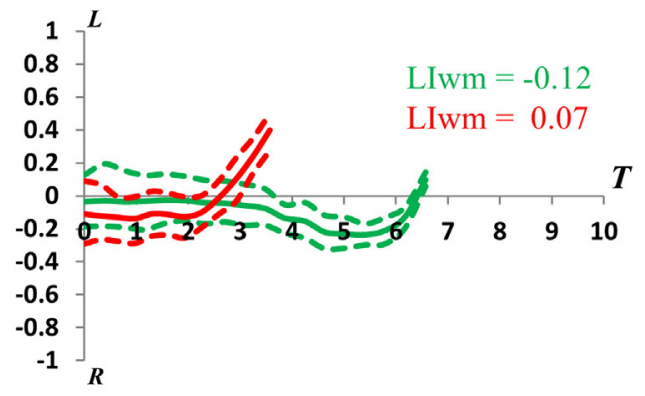

face responsive aIT

face selective aIT

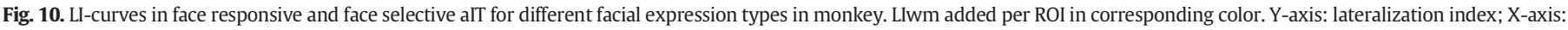
thresholded t-value. $\mathrm{L}=$ Left-sided, $\mathrm{R}=$ right-sided. $\mathrm{Scr}=$ Scrambled versions.

faces. Inter-individual differences in development (Dundas et al., 2013) or object processing strategies may play a role as well.

There are some methodological issues that need to be discussed with respect to lateralization in fMRI data. Traditionally a laterality index (LI) or statistical lateralization maps (Liegeois et al., 2002) have been used. Here we chose a bootstrapping method to calculate a weighted lateralization index (Wilke and Schmithorst, 2006). This robust method has the advantage to be threshold-independent, which allows plotting of different LIs at different thresholds, and minimizes the influence of statistical outliers (see also Matsuo et al. (2012) for a comparison of different LI calculation methods). Another issue using an LI is the choice of cut-off values defining lateralization. Although theoretical arguments can be made in favor of specific values, where a value of $+/-0.3$ could already be seen as a reasonable cut-off value, this still remains arbitrary (Seghier, 2008). We adopted a rather conservative approach by making a differentiation between weak lateralization and strong lateralization as used in previous studies (Pujol et al., 1999; Lehericy et al., 2000; Kosla et al., 2012) with cut-offs of 0.25 and 0.50 , respectively. Lower cut-off values that occur in the literature mainly pertain to presurgical studies assessing hemispheric dominance for language in refractory epilepsy patients (Springer et al., 1999; Sabsevitz et al., 2003; Suarez et al., 2009). Furthermore we used voxel values instead of voxel count in our calculations which allows a more subtle calculation.

Although we conclude that there is no lateralization for dynamic face processing in monkeys some results might suggest otherwise. Within monkey anterior inferotemporal cortex, lateralization curves show steep increases for certain contrasts. Such sharply bending curves that only occur at high thresholds for face responsive aIT does not allow drawing firm conclusions. In fact, such effects represent the core reason to use LI curves ranging over increasing thresholds and not LIs at a predefined threshold. These potential artifacts are more likely to occur when a small number of voxels in each hemisphere are used to calculate the LI with a high voxel value in one hemisphere. This would also seem to account for steep curves in face selective aIT where there is less activation for dynamic face responsive contrasts such that LI values are driven by a more limited number of voxels as voxel count drops quickly in both hemispheres. Logically, the risk for such effect is also proportional to the size of the ROI because the LI-calculating algorithm aborts when less than $10 \%$ of voxels are present in any hemisphere rendering larger ROIs more resistant. Moreover, the laterality effect in anterior inferotemporal cortex was not present for all examined contrasts nor was the direction of this effect congruent, discarding a consistent pattern of lateralization.

A limitation of the current study is the number of monkey subjects that were included $(n=3)$ compared to human subjects $(n=20)$.
Another limitation is that we studied speech stimuli in human subjects but not in monkeys. Accordingly, our comparative claims do not extend beyond non-verbal facial expressions. At the start of the study, we decided to present the same expressions from human and monkey faces to both species, and a monkey equivalent for speech expressions is less trivial to obtain than chewing and fearful expressions. It has been shown that the monkey STS is sensitive to rhythmic facial gestures within frequency range of audiovisual speech (Ghazanfar et al., 2010) and is involved, together with auditory cortex, in face and voice integration (Chandrasekaran and Ghazanfar, 2009). Although no laterality effects have been reported using dynamic rhythmic facial gestures in monkeys, it would be interesting to investigate this further.

To conclude, we found evidence for lateralization for dynamic face processing in humans but not in monkeys. Lateralization was present in superior temporal sulcus but not in ventral occipitotemporal cortex, pleading for a regional neural reorganization during human or hominoid evolution. Finally the laterality pattern was expression-dependent and differed between verbal and non-verbal expressions. Taken together, we speculate that the development of the left-hemispheric language network contributed critically to the functional brain asymmetry that we observed in humans for dynamic face processing.

Supplementary data to this article can be found online at http://dx. doi.org/10.1016/j.neuroimage.2014.11.020.

\section{Acknowledgments}

We thank C. Fransen, C. Van Eupen and A. Coeman for animal training and care; and D. Mantini, H. Kolster, W. Depuydt, G. Meulemans, P. Kayenbergh, M. De Paep, S. Verstraeten, and I. Puttemans for technical assistance. This work was supported by the Fund for Scientific Research (Flanders) G043912, G074609, G062208, and G083111, Hercules II Funding, Inter University Attraction Pole 7/11. Q.Z. and J.V.d.S. are postdoctoral fellows of the FWO Vlaanderen.

\section{References}

Bernstein, L.E., Jiang, J., Pantazis, D., Lu, Z.L., Joshi, A., 2011. Visual phonetic processing localized using speech and nonspeech face gestures in video and point-light displays. Hum. Brain Mapp. 32, 1660-1676.

Broman, M., 1978. Reaction-time differences between the left and right hemispheres for face and letter discrimination in children and adults. Cortex 14, 578-591.

Bukowski, H., Dricot, L., Hanseeuw, B., Rossion, B., 2013. Cerebral lateralization of facesensitive areas in left-handers: only the FFA does not get it right. Cortex 49, 2583-2589.

Busigny, T., Joubert, S., Felician, O., Ceccaldi, M., Rossion, B., 2010. Holistic perception of the individual face is specific and necessary: evidence from an extensive case study of acquired prosopagnosia. Neuropsychologia 48, 4057-4092. 
Calvert, G.A., Bullmore, E.T., Brammer, M.J., Campbell, R., Williams, S.C., McGuire, P.K., Woodruff, P.W., Iversen, S.D., David, A.S., 1997. Activation of auditory cortex during silent lipreading. Science 276, 593-596.

Calvert, G.A., Campbell, R., Brammer, M.J., 2000. Evidence from functional magnetic resonance imaging of crossmodal binding in the human heteromodal cortex. Curr. Biol. 10, 649-657.

Campbell, R., MacSweeney, M., Surguladze, S., Calvert, G., McGuire, P., Suckling, J., Brammer, M.J., David, A.S., 2001. Cortical substrates for the perception of face actions: an fMRI study of the specificity of activation for seen speech and for meaningless lower-face acts (gurning). Brain Res. Cogn. Brain Res. 12, 233-243.

Chandrasekaran, C., Ghazanfar, A.A., 2009. Different neural frequency bands integrate faces and voices differently in the superior temporal sulcus. J. Neurophysiol. 101, 773-788.

Corballis, P.M., 2003. Visuospatial processing and the right-hemisphere interpreter. Brain Cogn. 53, 171-176.

Corballis, P.M., Funnell, M.G., Gazzaniga, M.S., 2000. An evolutionary perspective on hemispheric asymmetries. Brain Cogn. 43, 112-117.

Dahl, C.D., Rasch, M.J., Tomonaga, M., Adachi, I., 2013. Laterality effect for faces in chimpanzees (Pan troglodytes). J. Neurosci. 33, 13344-13349.

de Gelder, B., Van den Stock, J., 2010. Moving and being moved. The relative importance of dynamical information for residual face processing in clinical populations and brain damaged patients. In: Curio, C., Bülthoff, H.H., Giese, M.A. (Eds.), Dynamic Faces: Insights from Experiments and Computation. MIT Press, pp. 161-173.

De Renzi, E., Perani, D., Carlesimo, G.A., Silveri, M.C., Fazio, F., 1994. Prosopagnosia can be associated with damage confined to the right hemisphere-an MRI and PET study and a review of the literature. Neuropsychologia 32, 893-902.

Dien, J., 2009. A tale of two recognition systems: implications of the fusiform face area and the visual word form area for lateralized object recognition models. Neuropsychologia 47, 1-16.

Dundas, E.M., Plaut, D.C., Behrmann, M., 2013. The joint development of hemispheric lateralization for words and faces. J. Exp. Psychol. Gen. 142, 348-358.

Ellis, H.D., Shepherd, J.W., 1975. Recognition of upright and inverted faces presented in the left and right visual fields. Cortex 11,3-7.

Fitch, W.T., Huber, L., Bugnyar, T., 2010. Social cognition and the evolution of language: constructing cognitive phylogenies. Neuron 65, 795-814.

Foley, E., Rippon, G., Thai, N.J., Longe, O., Senior, C., 2012. Dynamic facial expressions evoke distinct activation in the face perception network: a connectivity analysis study. J. Cogn. Neurosci. 24, 507-520.

Fusar-Poli, P., Placentino, A., Carletti, F., Landi, P., Allen, P., Surguladze, S., Benedetti, F. Abbamonte, M., Gasparotti, R., Barale, F., Perez, J., McGuire, P., Politi, P., 2009. Functional atlas of emotional faces processing: a voxel-based meta-analysis of 105 functional magnetic resonance imaging studies. J. Psychiatry Neurosci. 34, 418-432.

Ghazanfar, A.A., Chandrasekaran, C., Morrill, R.J., 2010. Dynamic, rhythmic facial expressions and the superior temporal sulcus of macaque monkeys: implications for the evolution of audiovisual speech. Eur. J. Neurosci. 31, 1807-1817.

Gschwind, M., Pourtois, G., Schwartz, S., Van De Ville, D., Vuilleumier, P., 2012. Whitematter connectivity between face-responsive regions in the human brain. Cereb. Cortex 22, 1564-1576.

Hall, D.A., Fussell, C., Summerfield, A.Q., 2005. Reading fluent speech from talking faces: typical brain networks and individual differences. J. Cogn. Neurosci. 17, 939-953.

Hamilton, C.R., Vermeire, B.A., 1988. Complementary hemispheric specialization in monkeys. Science 242, 1691-1694.

Haxby, J.V., Ungerleider, L.G., Clark, V.P., Schouten, J.L., Hoffman, E.A., Martin, A., 1999. The effect of face inversion on activity in human neural systems for face and object perception. Neuron 22, 189-199.

Ishai, A., Haxby, J.V., Ungerleider, L.G., 2002. Visual imagery of famous faces: effects of memory and attention revealed by fMRI. Neurolmage 17, 1729-1741.

Janssens, T., Zhu, Q., Popivanov, I.D., Vanduffel, W., 2014. Probabilistic and single-subject retinotopic maps reveal the topographic organization of face patches in the macaque cortex. J. Neurosci. 34, 10156-10167.

Jason, G.W., Cowey, A., Weiskrantz, L., 1984. Hemispheric asymmetry for a visuo-spatial task in monkeys. Neuropsychologia 22, 777-784.

Kanwisher, N., Barton, J.J.S., 2011. The functional architecture of the face system: integrating evidence from fMRI and patient studies. In: Calder, A.J., Rhodes, G., Johnson, M.H. Haxby, J.V. (Eds.), The Oxford Handbook of Face Perception. Oxford University Press, New York, pp. 111-129.

Kanwisher, N., McDermott, J., Chun, M.M., 1997. The fusiform face area: a module in human extrastriate cortex specialized for face perception. J. Neurosci. 17, 4302-4311.

Knecht, S., Drager, B., Deppe, M., Bobe, L., Lohmann, H., Floel, A., Ringelstein, E.B. Henningsen, H., 2000. Handedness and hemispheric language dominance in healthy humans. Brain 123 (Pt 12), 2512-2518.

Kolster, H., Mandeville, J.B., Arsenault, J.T., Ekstrom, L.B., Wald, L.L., Vanduffel, W., 2009. Visual field map clusters in macaque extrastriate visual cortex. J. Neurosci. 29, 7031-7039.

Kosla, K., Pfajfer, L., Bryszewski, B., Jaskolski, D., Stefanczyk, L., Majos, A., 2012. Functional rearrangement of language areas in patients with tumors of the central nervous system using functional magnetic resonance imaging. Pol. J. Radiol./Pol. Med. Soc. Radiol. 77, 39-45.

LaBar, K.S., Crupain, M.J., Voyvodic, J.T., McCarthy, G., 2003. Dynamic perception of facia affect and identity in the human brain. Cereb. Cortex 13, 1023-1033.

Lehericy, S., Cohen, L., Bazin, B., Samson, S., Giacomini, E., Rougetet, R., Hertz-Pannier, L. Le Bihan, D., Marsault, C., Baulac, M., 2000. Functional MR evaluation of tempora and frontal language dominance compared with the Wada test. Neurology 54 $1625-1633$.

Liegeois, F., Connelly, A., Salmond, C.H., Gadian, D.G., Vargha-Khadem, F., Baldeweg, T. 2002. A direct test for lateralization of language activation using fMRI: comparison with invasive assessments in children with epilepsy. Neurolmage 17, 1861-1867.
MacSweeney, M., Calvert, G.A., Campbell, R., McGuire, P.K., David, A.S., Williams, S.C., Woll, B., Brammer, M.J., 2002. Speechreading circuits in people born deaf. Neuropsychologia 40, 801-807.

Matsuo, K., Chen, S.H., Tseng, W.Y., 2012. AveLI: a robust lateralization index in functional magnetic resonance imaging using unbiased threshold-free computation. J. Neurosci. Methods 205, 119-129.

McLaren, D.G., Kosmatka, K.J., Oakes, T.R., Kroenke, C.D., Kohama, S.G., Matochik, J.A., Ingram, D.K., Johnson, S.C., 2009. A population-average MRI-based atlas collection of the rhesus macaque. Neurolmage 45, 52-59.

Meng, M., Cherian, T., Singal, G., Sinha, P., 2012. Lateralization of face processing in the human brain. Proc. Biol. Sci. 279, 2052-2061.

Milner, B., 1971. Interhemispheric differences in the localization of psychological processes in man. Br. Med. Bull. 27, 272-277.

Moeller, S., Freiwald, W.A., Tsao, D.Y., 2008. Patches with links: a unified system for processing faces in the macaque temporal lobe. Science 320, 1355-1359.

Morris, R.D., Hopkins, W.D., 1993. Perception of human chimeric faces by chimpanzees: evidence for a right hemisphere advantage. Brain Cogn. 21, 111-122.

Noesselt, T., Driver, J., Heinze, H.J., Dolan, R., 2005. Asymmetrical activation in the human brain during processing of fearful faces. Curr. Biol. 15, 424-429.

Overman Jr., W.H., Doty, R.W., 1982. Hemispheric specialization displayed by man but not macaques for analysis of faces. Neuropsychologia 20, 113-128.

Papenberg, N., Bruhn, A., Brox, T., Didas, S., Weickert, J., 2006. Highly accurate optic flow computation with theoretically justified warping. Int. J. Comput. Vis. 67, 141-158.

Paulesu, E., Perani, D., Blasi, V., Silani, G., Borghese, N.A., De Giovanni, U., Sensolo, S., Fazio, F., 2003. A functional-anatomical model for lipreading. J. Neurophysiol. 90, 2005-2013.

Pinsk, M.A., Arcaro, M., Weiner, K.S., Kalkus, J.F., Inati, S.J., Gross, C.G., Kastner, S., 2009. Neural representations of faces and body parts in macaque and human cortex: a comparative FMRI study. J. Neurophysiol. 101, 2581-2600.

Pitcher, D., Dilks, D.D., Saxe, R.R., Triantafyllou, C., Kanwisher, N., 2011. Differential selectivity for dynamic versus static information in face-selective cortical regions. Neurolmage 56, 2356-2363.

Plotnik, J., Nelson, P.A., de Waal, F.B., 2003. Visual field information in the face perception of chimpanzees (Pan troglodytes). Ann. N. Y. Acad. Sci. 1000, 94-98.

Polosecki, P., Moeller, S., Schweers, N., Romanski, L.M., Tsao, D.Y., Freiwald, W.A., 2013. Faces in motion: selectivity of macaque and human face processing areas for dynamic stimuli. J. Neurosci. 33, 11768-11773.

Popivanov, I.D., Jastorff, J., Vanduffel, W., Vogels, R., 2012. Stimulus representations in body-selective regions of the macaque cortex assessed with event-related fMRI. Neurolmage 63, 723-741.

Pujol, J., Deus, J., Losilla, J.M., Capdevila, A., 1999. Cerebral lateralization of language in normal left-handed people studied by functional MRI. Neurology 52, 1038-1043.

Reynolds, D.M., Jeeves, M.A., 1978. A developmental study of hemisphere specialization for recognition of faces in normal subjects. Cortex 14, 511-520.

Sabsevitz, D.S., Swanson, S.J., Hammeke, T.A., Spanaki, M.V., Possing, E.T., Morris 3rd, G.L., Mueller, W.M., Binder, J.R., 2003. Use of preoperative functional neuroimaging to predict language deficits from epilepsy surgery. Neurology 60, 1788-1792.

Sato, W., Kochiyama, T., Yoshikawa, S., Naito, E., Matsumura, M., 2004. Enhanced neural activity in response to dynamic facial expressions of emotion: an fMRI study. Brain Res. Cogn. Brain Res. 20, 81-91.

Seghier, M.L., 2008. Laterality index in functional MRI: methodological issues. Magn. Reson. Imaging 26, 594-601.

Sergent, J., Signoret, J.L., 1992. Varieties of functional deficits in prosopagnosia. Cereb. Cortex 2, 375-388.

Springer, J.A., Binder, J.R., Hammeke, T.A., Swanson, S.J., Frost, J.A., Bellgowan, P.S., Brewer, C.C., Perry, H.M., Morris, G.L., Mueller, W.M., 1999. Language dominance in neurologically normal and epilepsy subjects: a functional MRI study. Brain 122 (Pt 11), 2033-2046.

Suarez, R.O., Whalen, S., Nelson, A.P., Tie, Y., Meadows, M.E., Radmanesh, A., Golby, A.J., 2009. Threshold-independent functional MRI determination of language dominance: a validation study against clinical gold standards. Epilepsy Behav. 16, 288-297.

Tong, F., Nakayama, K., Moscovitch, M., Weinrib, O., Kanwisher, N., 2000. Response properties of the human fusiform face area. Cogn. Neuropsychol. 17, 257-280.

Tsao, D.Y., Freiwald, W.A., Knutsen, T.A., Mandeville, J.B., Tootell, R.B., 2003. Faces and objects in macaque cerebral cortex. Nat. Neurosci. 6, 989-995.

Vallortigara, G., Rogers, L.J., Bisazza, A., 1999. Possible evolutionary origins of cognitive brain lateralization. Brain Res. Brain Res. Rev. 30, 164-175

Vandenbulcke, M., Peeters, R., Dupont, P., Van Hecke, P., Vandenberghe, R., 2007. Word reading and posterior temporal dysfunction in amnestic mild cognitive impairment. Cereb. Cortex 17, 542-551

Vermeire, B.A., Hamilton, C.R., 1998. Effects of facial identity, facial expression, and subject's sex on laterality in monkeys. Laterality 3, 1-19.

Wager, T.D., Nichols, T.E., 2003. Optimization of experimental design in fMRI: a general framework using a genetic algorithm. Neurolmage 18, 293-309.

Wilke, M., Lidzba, K., 2007. LI-tool: a new toolbox to assess lateralization in functional MR-data. J. Neurosci. Methods 163, 128-136.

Wilke, M., Schmithorst, V.J., 2006. A combined bootstrap/histogram analysis approach for computing a lateralization index from neuroimaging data. Neurolmage 33, 522-530.

Wilkinson, D., Ko, P., Wiriadjaja, A., Kilduff, P., McGlinchey, R., Milberg, W., 2009. Unilateral damage to the right cerebral hemisphere disrupts the apprehension of whole faces and their component parts. Neuropsychologia 47, 1701-1711.

Zangenehpour, S., Chaudhuri, A., 2005. Patchy organization and asymmetric distribution of the neural correlates of face processing in monkey inferotemporal cortex. Curr. Biol. 15, 993-1005.

Zhu, Q., Nelissen, K., den Stock, J.V., De Winter, F.L., Pauwels, K., de Gelder, B., Vanduffel, W., Vandenbulcke, M., 2013. Dissimilar processing of emotional facial expressions in human and monkey temporal cortex. Neurolmage 66C, 402-411. 